\title{
Shared biological risks that influence brain and behaviour
}

\author{
Miriam Cooper, ${ }^{1,2}$ Anita Thapar ${ }^{1,2}$
}

Attention deficit hyperactivity disorder (ADHD) is a common childhoodonset neurodevelopmental disorder. Comorbidity with other neurodevelopmental conditions, learning problems and psychiatric disorders is high and $\mathrm{ADHD}$ symptoms themselves, particularly inattention, can also persist into adulthood. ADHD is indexed by high levels of functional impairment and adversely affects social relationships, academic achievements and employment record. Rates of substance misuse and smoking behaviours are elevated, contributing to the physical and mental health disadvantage of those with ADHD.

Although highly heritable, $\mathrm{ADHD}$ is aetiologically complex whereby the coaction and interaction of inherited and noninherited factors likely play a role in its development, maintenance and adverse consequences. Indeed, no single causal risk factor, genetic or otherwise, has been identified to date as is the case for all common, complex disorders. The evidence suggests there is contribution from both common genetic variants of small effect size and rare variants (copy number variants) of larger effect size. ${ }^{1}$ Such variants have been found to impact on similar biological pathways, interestingly including one related to nicotinic acetylcholine receptor signalling. ${ }^{2}$ There is also growing evidence that $\mathrm{ADHD}$ inherited risks and associated gene variants have different phenotypic effects (pleiotropy). That is, the same set of genetic risks appears to influence $\mathrm{ADHD}$,

\section{(6) \\ OPEN ACCESS}

\footnotetext{
${ }^{1}$ Child \& Adolescent Psychiatry Section, Institute of Psychological Medicine and Clinical Neurosciences, Cardiff University School of Medicine, Cardiff, UK

${ }^{2}$ MRC Centre for Neuropsychiatric Genetics and Genomics, Cardiff University School of Medicine, Cardiff, UK
}

Correspondence to Dr Miriam Cooper,

Child \& Adolescent Psychiatry Section, Institute of Psychological Medicine and Clinical Neurosciences; Cardiff University School of Medicine, Heath Park, Cardiff CF14 4XN, UK; CooperML1@cardiff.ac.uk autism and other forms of developmental disorder/psychopathology and behaviour. A research question related to this observation is tackled by Thakur and colleagues. ${ }^{4}$

Their study sets out to examine genetic risks that may be shared between $\mathrm{ADHD}$ and the known comorbidity of smoking: a strategy which is attractive in view of $\mathrm{ADHD}$ being a risk factor for cigarette smoking and possible involvement of nicotinic acetylcholine receptor signalling. Also, it is a feasible approach given the genome-wide significant associations found in the large genome-wide meta-analyses of smoking behaviours. ${ }^{5}$ Exploration of shared aetiologies to try and unravel the complex neurobiology of $\mathrm{ADHD}$ is currently topical: there is growing research into shared biological and cognitive risk mechanisms between $\mathrm{ADHD}$ and co-occurring conditions such as autism using genetic, cognitive and imaging designs.

Another dimension to the strategy of Thakur et $a l^{4}$ is an interesting finding that the authors highlight: that $\mathrm{ADHD}$ has consistently been found to be associated with exposure to maternal smoking during pregnancy. This has been thought to be due to the causal risk effects of cigarette smoke on the developing foetus as there are biologically plausible mechanisms. The constituents of cigarette smoke can alter physiological processes which might in turn contribute to aberrant neurodevelopment in $\mathrm{ADHD}$. However, research suggests that the association with maternal smoking during pregnancy may be due to the confounding effects of shared genetic liability between maternal smoking behaviour and offspring $\mathrm{ADHD}{ }^{6}$

The study by Thakur et $a l^{4}$ benefits from a strong theoretical premise and hypothesis-driven approach and detailed neurocognitive profiling and rigorous phenotypic description with a dimensional and categorical approach. In summary, the research finds that of the five single nucleotide polymorphisms (SNPs) that were selected and considered to be significantly associated with smoking behaviour in the genome-wide meta-analysis, one, the $C^{*}$ allele of rs1329650 on 10q25 (which is associated with number of cigarettes smoked per day), is overtransmitted to the offspring with $\mathrm{ADHD}$ and is associated with several indices of phenotypic severity and neurocognitive dysfunction.

However, although the findings are potentially exciting, the authors are right to be tentative about their conclusions at this stage as there are certain cautions to be exercised when considering the implications. At a conceptual level, the SNPs found to reach genome-wide significance in the smoking meta-analysis have not been found to be associated with $\mathrm{ADHD}$ in either $\mathrm{ADHD}$ candidate gene metaanalyses or a meta-analysis of $\mathrm{ADHD}$ genome-wide association studies (GWAS). With small sample sizes (a limitation of all $\mathrm{ADHD}$ genetic studies to date) and the small effect sizes of individual gene variants, results can reflect Type 1 or Type 2 errors. Replication, as the authors state, is always necessary-especially as multiple outcomes were tested.

On the other hand, it is worth bearing in mind that studies investigating $\mathrm{ADHD}$ genetics to date have not had sample sizes comparable with those that have had success for other complex disorders, including psychiatric disorders. The meta-analysis of ADHD GWAS, which involves hypothesis-free testing of thousands of genetic variants, is almost certainly underpowered to detect effects. It may be that the common gene variants examined in the study by Thakur et al are found, in time, to be significant in subsequent GWAS or meta-analyses of larger $\mathrm{ADHD}$ samples. Such replications would lend greater weight to the results of this current investigation.

This interesting and novel study should be interpreted as preliminary evidence that the theoretical plausibility of shared inherited risks underlying $\mathrm{ADHD}$ and smoking behaviours could extend to detecting shared gene variants at a molecular level (see figure 1). Future research could perhaps consider replicating findings using case control designs as well as considering different ways of capturing smoking-associated genetic variation. It would also be interesting to investigate longitudinally the effects of smoking associated gene variants including the SNPs in this study, and to test whether they contribute to the known elevated risk of smoking behaviour in those with $\mathrm{ADHD}$.

In summary, although the results of this exploratory study can only be considered as preliminary findings at present, this is an intriguing starting point from which to conduct further related 

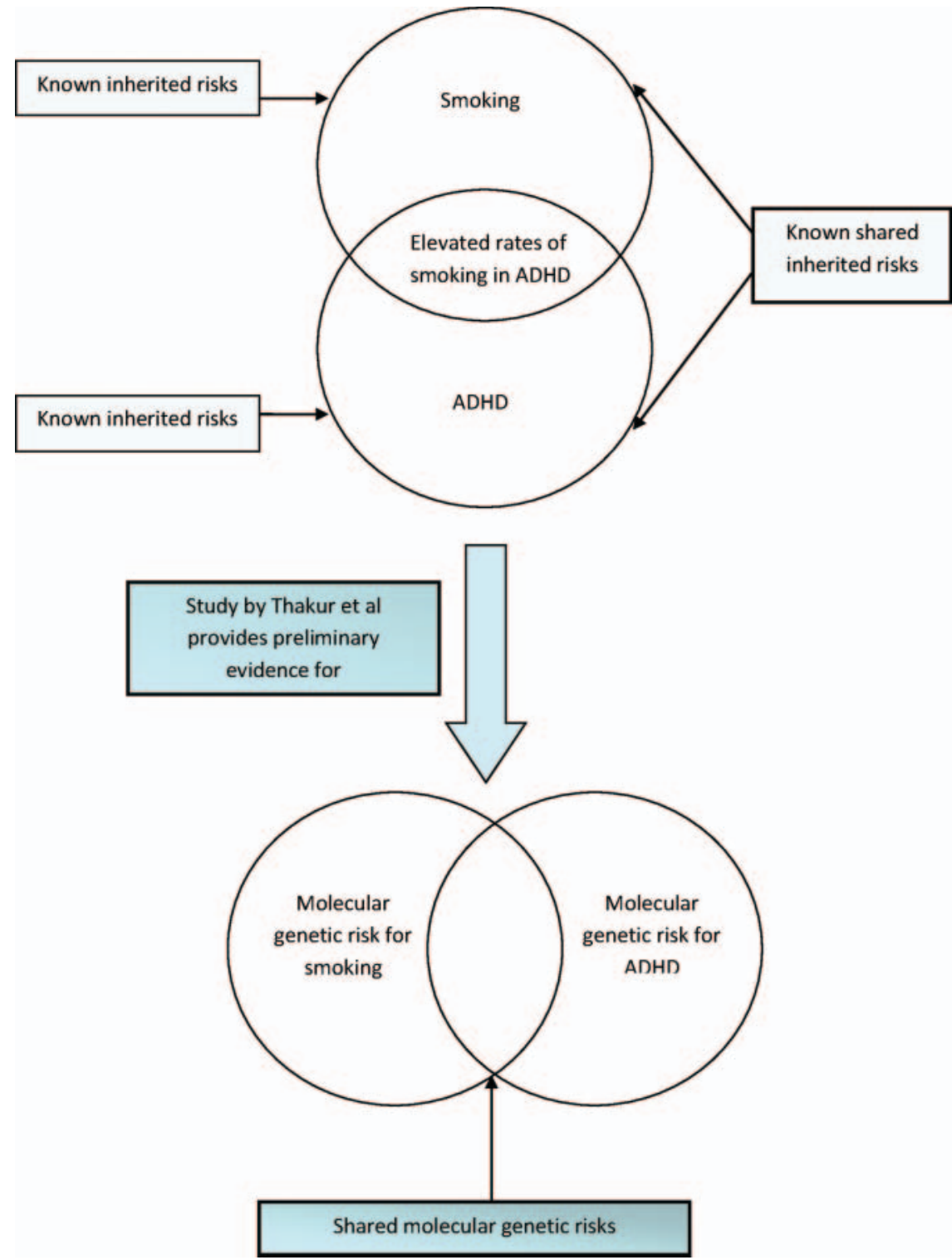

Figure 1 Illustration of shared genetic risks between attention deficit hyperactivity disorder (ADHD) and smoking. analyses. Acknowledging that the same genetic risk variants can have different phenotypic effects could help inform discovery of risk variants for childhood developmental/psychiatric disorders for which it can be difficult to assemble very large sample sizes. Such efforts could help uncover novel biological risk pathways and contribute to explaining neurodevelopmental comorbidities.

Funding MRC and Wellcome Trust.

Competing interests None.

Provenance and peer review Commissioned; externally peer reviewed.

Accepted 6 August 2012

Published Online First 29 October 2012

Arch Dis Child 2012:97:1011-1012.

doi:10.1136/archdischild-2012-302461

\section{REFERENCES}

1. Thapar A, Cooper M, Jefferies R, et al. What causes attention deficit hyperactivity disorder? Arch Dis Child 2012;97:260-5.

2. Stergiakouli $\mathbf{E}$, Hamshere $\mathrm{M}$, Holmans $\mathrm{P}$, et al. Investigating the contribution of common genetic variants to the risk and pathogenesis of ADHD. Am J Psychiatry 2012;169:186-94.

3. Williams NM, Franke B, Mick E, et al. Genome-wide analysis of copy number variants in attention deficit hyperactivity disorder: the role of rare variants and duplications at 15q13.3. Am J Psychiatry 2012;169:195-204.

4. Joober J, Thakur GA, Sengupta SM, et al. Family-based association study of ADHD and genes increasing the risk for smoking behaviors. Arch Dis Child 2012;97:1027-33.

5. Tobacco and Genetics Consortium. Genome-wide meta-analyses identify multiple loci associated with smoking behavior. Nat Genet 2010;42:441-7.

6. Thapar A, Rice F, Hay D, et al. Prenatal smoking might not cause attention-deficit/hyperactivity disorder: evidence from a novel design. Biol Psychiatry 2009;66:722-7. 